\title{
Emergence of a mupirocin-resistant, methicillin-susceptible Staphylococcus aureus clone associated with skin and soft tissue infections in Greece
}

\author{
Nikolaos Giormezis ${ }^{1,2}$, Anastassios Doudoulakakis ${ }^{3}$, Katerina Tsilipounidaki $^{4}$, Maria Militsopoulou $^{5}$, \\ George Kalogeras ${ }^{3}$, Vasiliki Stamouli ${ }^{5}$, Fevronia Kolonitsiou' ${ }^{1}$, Efthimia Petinaki ${ }^{4}$, Evangelia Lebessi ${ }^{3}$ and \\ Iris Spiliopoulou ${ }^{1,2^{*}}$
}

\begin{abstract}
Background: Staphylococcus aureus causes various infections, including skin and soft tissue infections (SSTIs). In this study, methicillin-susceptible S. aureus (MSSA) from SSTls among patients in three tertiary-care hospitals in Greece were studied in terms of antimicrobial resistance, clonal distribution, toxin and adhesin genes carriage.

Results: During a five-year period (2014-2018), 6145 S. aureus were recovered from 13,244 patients with SSTIs and tested for antimicrobial susceptibility. MSSA were 4806 (78.21\%) including 1484 isolates with mupirocin minimum inhibitory concentration (MIC) > 64 mg/L (30.88\%). Two hundred and sixty representative mupirocin-resistant MSSA were analyzed for genes encoding Panton-Valentine leukocidin (PVL, lukS/lukF-PV), exfoliative toxins (eta, etb), adhesin FnbA (fnbA) and resistance genes mupA (high-level resistance to mupirocin), fusB (fusidic acid), aminoglycosides' modifying enzymes, ermA, ermC and msrA (macrolides/lincosamides) by PCRs. Strains were classified into clones by PFGE and MLST.

All mupirocin-resistant MSSA were penicillin-resistant; $92.7 \%$ expressed resistance to fusidic acid and $88.9 \%$ to tobramycin. All 260 molecularly analyzed isolates were mupA-positive; all fusidic acid-resistant (241/260) carried fusB whereas, the tobramycin-resistant ones (230), ant(4')-la. The majority carried eta $(93.85 \%)$, etb (98.08\%) and fnbA (88.85\%). PFGE typing revealed a mostly unvarying population; 260 MSSA were grouped into three types. One major eta/etb-positive clone comprising of 258/260 strains (99.2\%), PFGE type 1, was classified as ST121, including nine strains co-carrying PVL. Another PVL-positive strain was identified as ST1, and one toxins-negative as ST21.

Conclusions: A mupirocin-resistant MSSA clone, ST121, carrying resistance, exfoliative toxins and adhesin genes, was spread and predominated in SSTIs from patients in Greece during the five-year studied period.
\end{abstract}

Keywords: MSSA, SSTIs, Mupirocin-resistant, ST121, Exfoliative toxins, Greece

\footnotetext{
*Correspondence: spiliopl@upatras.gr

'Department of Microbiology, School of Medicine, University of Patras, 26504 Patras, Greece

${ }^{2}$ National Reference Laboratory for Staphylococci, University of Patras, Patras, Greece

Full list of author information is available at the end of the article
}

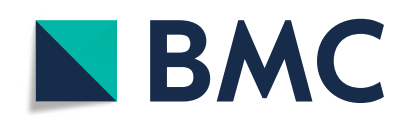

(- The Author(s). 2021 Open Access This article is licensed under a Creative Commons Attribution 4.0 International License, which permits use, sharing, adaptation, distribution and reproduction in any medium or format, as long as you give appropriate credit to the original author(s) and the source, provide a link to the Creative Commons licence, and indicate if changes were made. The images or other third party material in this article are included in the article's Creative Commons licence, unless indicated otherwise in a credit line to the material. If material is not included in the article's Creative Commons licence and your intended use is not permitted by statutory regulation or exceeds the permitted use, you will need to obtain permission directly from the copyright holder. To view a copy of this licence, visit http://creativecommons.org/licenses/by/4.0/. The Creative Commons Public Domain Dedication waiver (http://creativecommons.org/publicdomain/zero/1.0/) applies to the data made available in this article, unless otherwise stated in a credit line to the data. 


\section{Background}

Staphylococcus aureus is an extremely common bacterium in human population with around $20-30 \%$ of people being carriers, more frequently in the anterior nasal cavity, but also the skin, the pharynx and the gastrointestinal tract [1]. Despite being part of the human flora, staphylococci can cause a wide range of infections, including skin and soft tissue infections (SSTIs), pneumonia and bacteraemia [2].

Several factors contribute to the establishment of staphylococcal infections. The initial attachment of the microorganism is promoted by adhesins grouped into the single family named Microbial Surface Components Recognizing Adhesive Matrix Molecules (MSCRAMMs) [3]. The $S$. aureus fibronectin-binding protein A (FnbA), encoded by $f n b A$ gene, possesses multiple regions capable of conferring adherence to both soluble and immobilized forms of fibronectin [4]. This confers $S$. aureus the ability to invade endothelial cells in vivo and in vitro. Moreover, FnbA promotes bacterial attachment to fibrinogen as well as, adherence and aggregation of activated platelets [5]. Several studies have demonstrated the prevalence of FnbA against the homologous protein FnbB [6, 7].

Another important factor in the pathogenesis of staphylococcal infections is the production of exotoxins. Panton-Valentine leukocidin (PVL) is a cytotoxin produced by $S$. aureus that causes leukocyte destruction and tissue necrosis. The toxin is detected in large percentages among isolates that cause necrotic skin lesions and severe necrotizing pneumonia. Clinical and epidemiological studies suggest the involvement of PVL as an important factor contributing to the epidemic spread and virulence of communityacquired MRSA, however, this is still intensely debated [8]. The exfoliative toxins of S. aureus are responsible for the staphylococcal scalded skin syndrome (SSSS), a blistering skin disorder particularly affecting infants and young children, as well as adults with underlying disease. The target for the toxins has been identified as desmoglein-1, a desmosomal glycoprotein which plays an important role in maintaining cell-to-cell adhesion in the superficial epidermis. Exfoliative toxins $\mathrm{A}$ and $\mathrm{B}$ are the most frequently implicated in human skin damage [9].

Staphylococci express resistance to many antimicrobial agents, an increasing problem worldwide, especially among nosocomial pathogens. $S$. aureus is a leading cause of bacteremia and infective endocarditis as well as osteoarticular, skin and soft tissue, pleuropulmonary, and device-related infections [10]. From the first report on methicillin resistance in 1961 till today, methicillinresistant staphylococci (MRSA) represent a major health problem worldwide [11]. However, methicillin- susceptible $S$. aureus (MSSA) are also often the cause of infections.

Mupirocin is an antibiotic from monocarboxylic acid class used as antibacterial agent against $S$. aureus and can be obtained as a mixture of four pseudomonic acids by Pseudomonas fluorescens biosynthesis. It is used to control the MRSA outbreaks, SSTIs and nasal decolonization [12]. Due to its wide use without prescription, the microorganism's resistance to mupirocin increased from 1 to $81 \%$, thus compromising its place as therapeutic and decolonization agent [13]. In a previous study performed in a single pediatric hospital in Athens, Greece, a mupirocin-resistant ST121 clone among MSSA causing SSTIs was identified. This clone has emerged in 2013 and was detected to an increasing incidence till 2016 in this setting [14].

The observation that mupirocin-resistant MSSA were isolated with an increasing incidence since 2014 in two other tertiary care hospitals in Greece, led to the present study; MSSA with mupirocin minimum inhibitory concentration $(\mathrm{MIC})>1 \mathrm{mg} / \mathrm{L}$ recovered from SSTIs among patients admitted in three tertiary care hospitals in different areas of Greece (Athens, Southwestern and Central Greece) were studied in terms of antimicrobial resistance patterns, clonal distribution, toxins and adhesins gene carriage.

\section{Results}

From a total of 13,244 SSTIs recorded from 2014 to 2018, 6145 S. aureus were identified (46.40\%), recovered from different patients. Of them, 4806 isolates (78.21\%) were MSSA. All cefoxitin-susceptible isolates were also oxacillin-susceptible. An increase in the isolation rate of S. aureus from patients with SSTIs was detected from 2014 to 2016, followed by a decrease in the next two years (Table 1; Fig. 1). An increase of MSSA among $S$. aureus causing SSTIs was also observed during the fiveyear period, from $66.67 \%$ to 2014 to $88.15 \%$ in 2018 (Fig. 2).

One third of MSSA, 1484/4806 (30.88\%), associated with SSTIs showed mupirocin MICs ranging from $64 \mathrm{mg} / \mathrm{L}$ to $>1024 \mathrm{mg} / \mathrm{L}$. Most isolates (1423/1484, $95.9 \%$ ) exhibited high-level resistance (>512 $\mathrm{mg} / \mathrm{L}$ ) to mupirocin. From 2014 to 2017, an increase of mupirocin-resistant isolates among MSSA causing SSTIs was observed, from 16.45 to $38.66 \%$, followed by a decrease in 2018 (33.7\%) (Fig. 2). All mupirocin-resistant isolates were also penicillin-resistant, whereas, 1376/ 1484 were also resistant to fusidic acid (92.7\%), and 1320 to tobramycin (88.9\%) (Fig. 3). Three hundred and thirteen expressed resistance to erythromycin (21.1\%), out of which 283 were also clindamycin-resistant (184 exhibited constitutive and 99 inducible resistance). The majority of isolates was multi-resistant; however, all were 


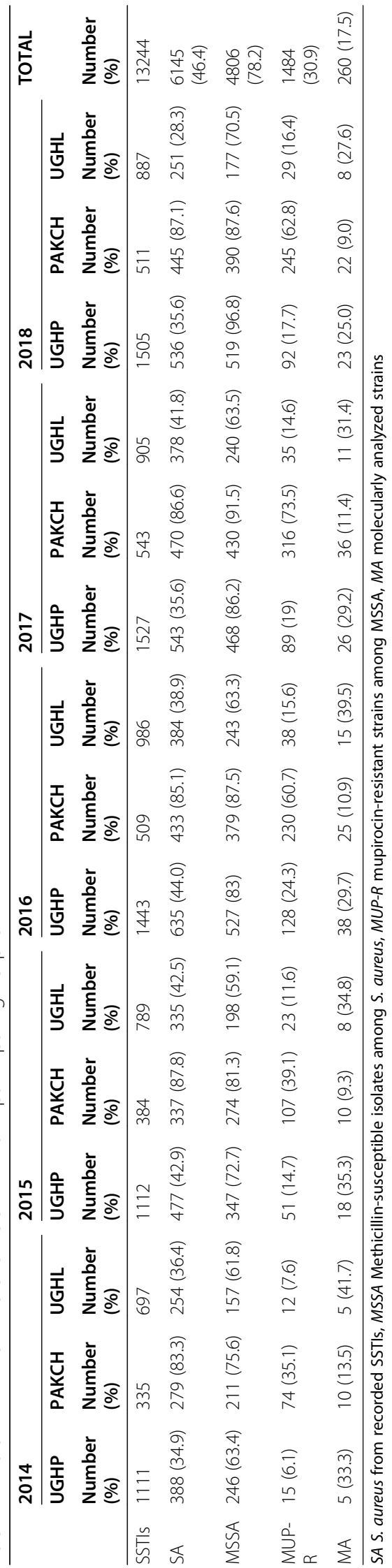




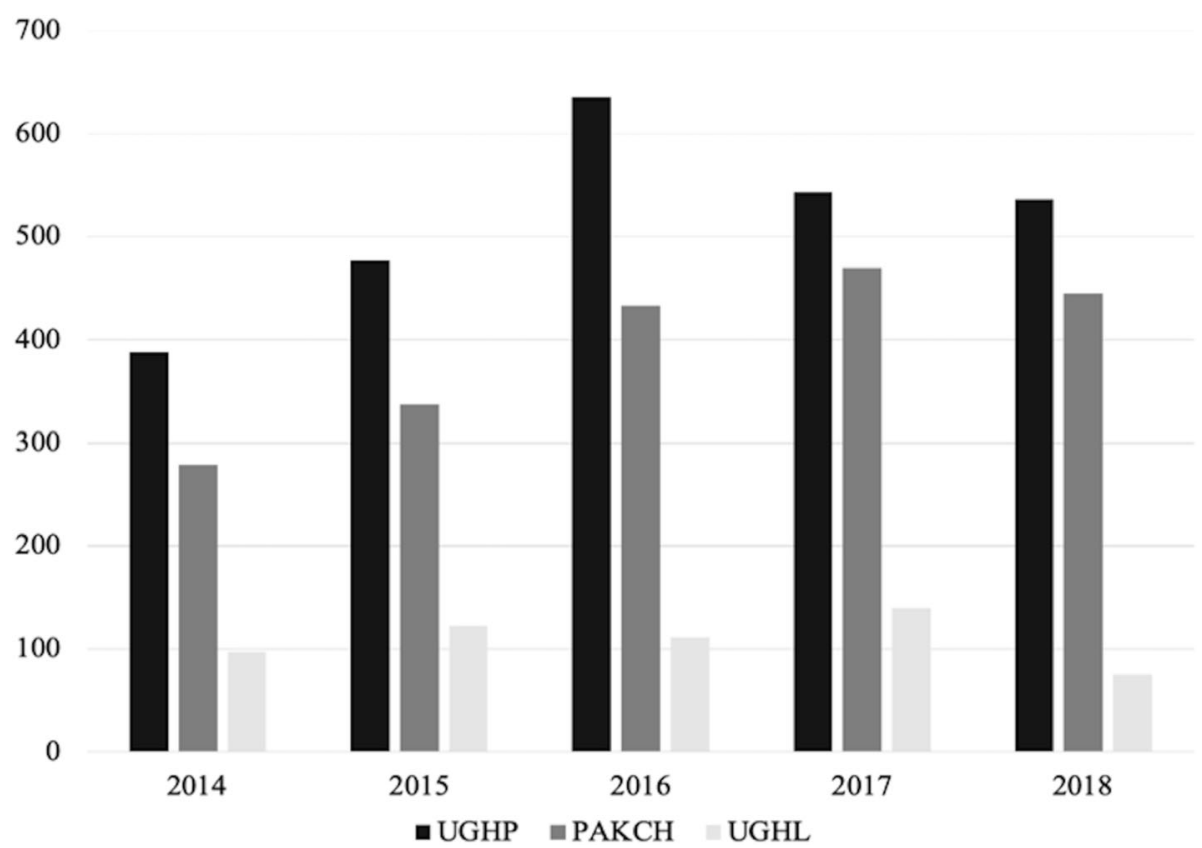

Fig. 1 Number of S. aureus isolates recovered from patients with SSTIS, during the studied period (2014-2018). University General Hospital of Patras: UGHP; P. \& A. Kyriakou Children's Hospital: PAKCH; University General Hospital of Larissa: UGHL)

susceptible to rifampicin and sulfamethoxazole/trimethoprim (Fig. 3).

From the total of 260 molecularly analyzed isolates, 181 were recovered from children and 79 from adults; all were mupA-positive, while all fusidic acid-resistant isolates $(241 / 260)$ carried fusB and the tobramycin- resistant ones (230/260) carried ant(4')-Ia. From the 55/ 260 erythromycin-resistant isolates, 50 were also resistant to clindamycin; $51 / 55$ carried erm $C$ and four the ermA gene. The five erythromycin-resistant/clindamycin-susceptible strains carried mrs $A$ gene encoding the MsrA efflux pump.

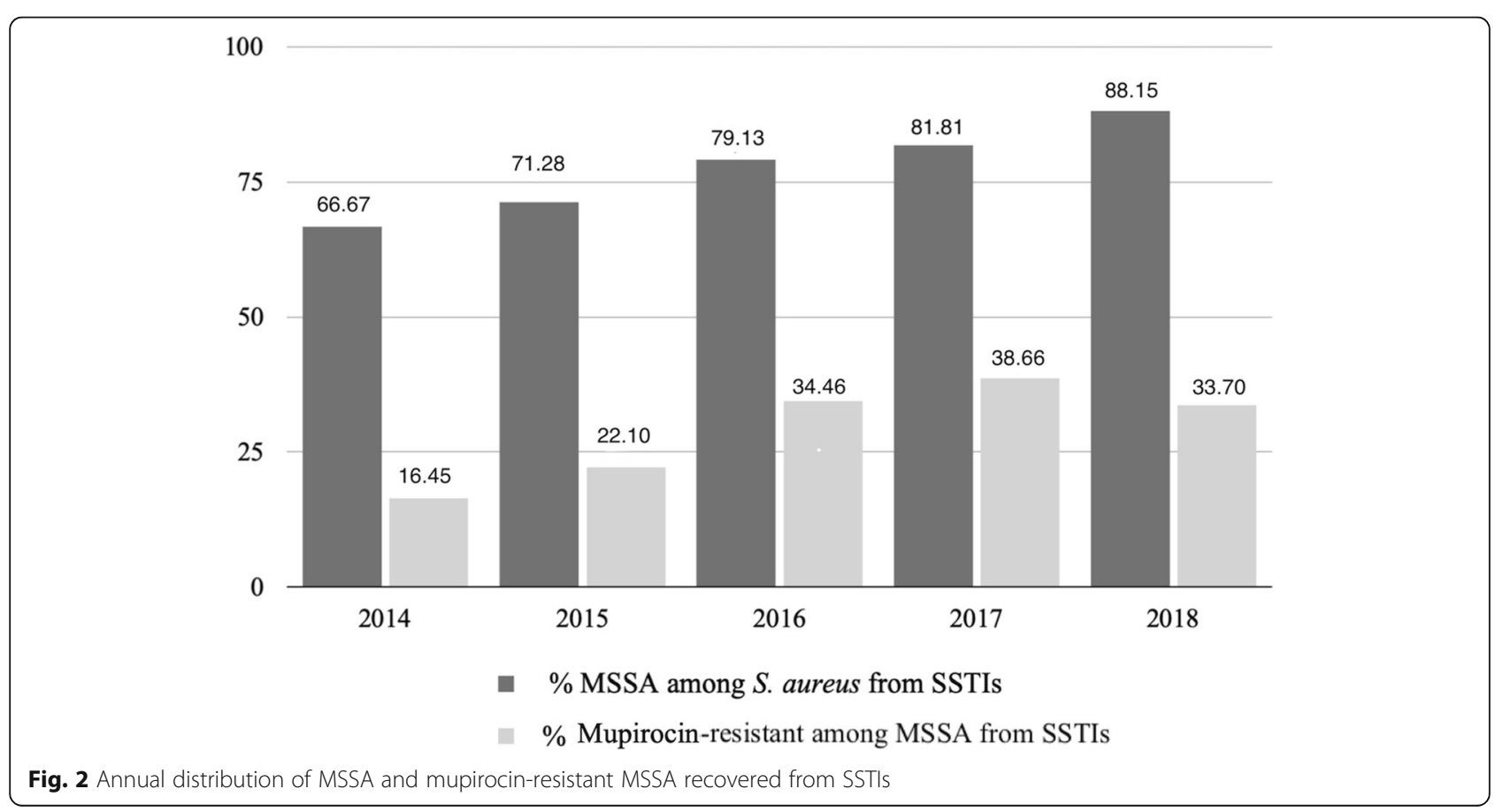




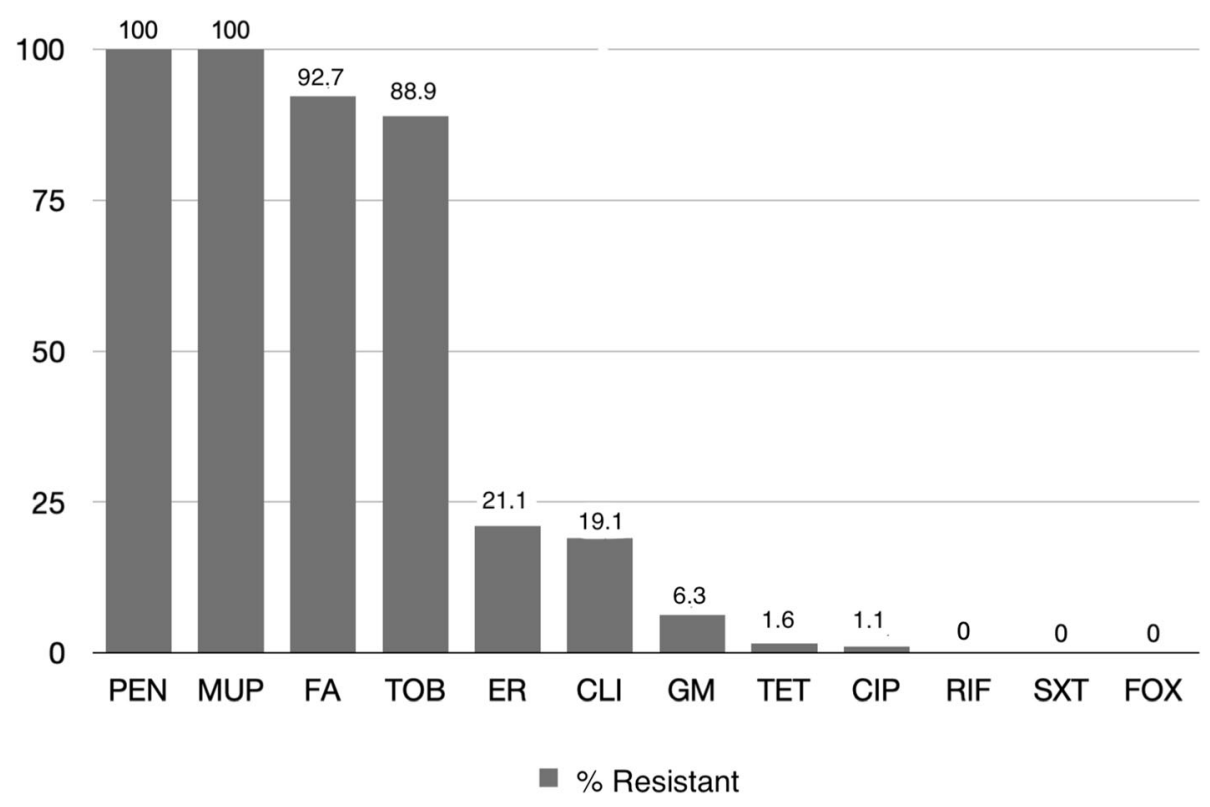

Fig. 3 Antimicrobial resistance patterns of mupirocin-resistant MSSA from SSTIs. Antimicrobials used: penicillin (PEN), mupirocin (MUP), fusidic acid (FA), tobramycin (TOB), erythromycin (ER), clindamycin (CLI), gentamicin (GM), tetracycline (TET), ciprofloxacin (CIP), rifampicin (RIF), sulfamethoxazole/trimethoprim (SXT), cefoxitin (FOX)

Dice (Tol 1.0\%-1.0\%) (H>0.0\% S>0.0\%) [0.0\%-100.0\%]

mssa1

mssa1

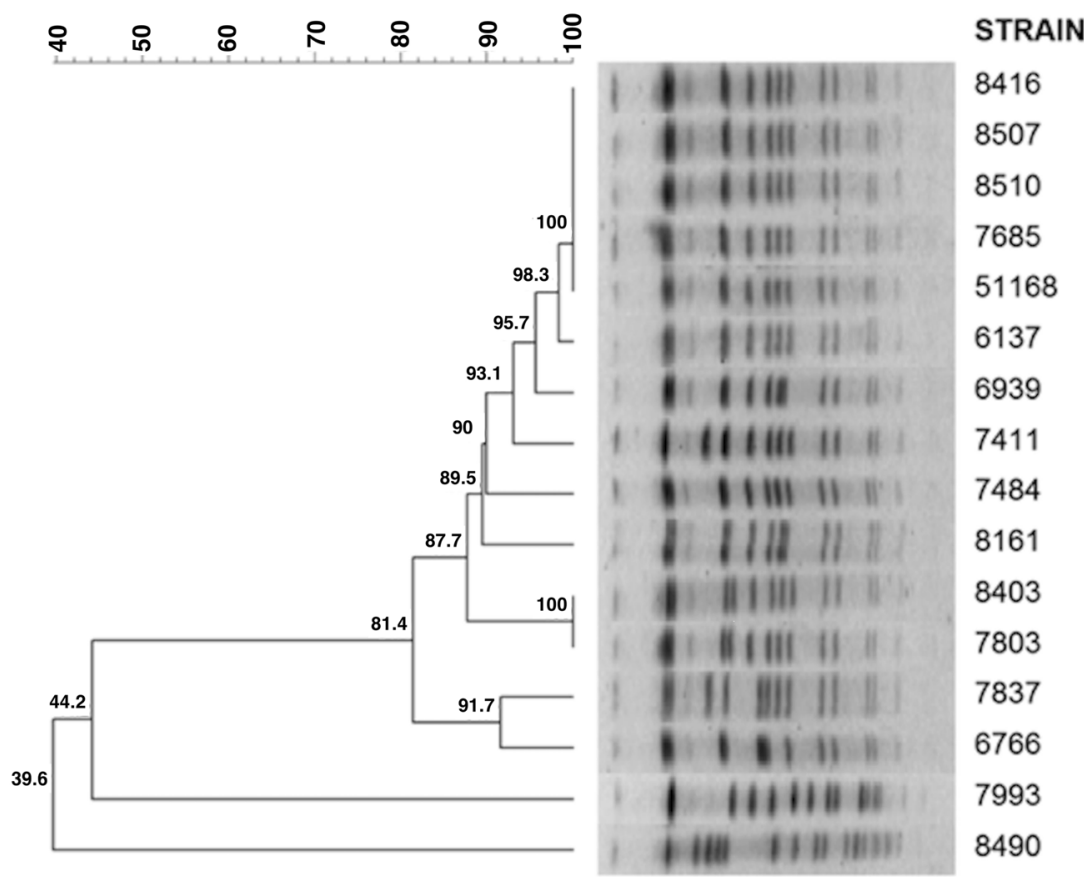

CITY
PATRAS
PATRAS
PATRAS
LARISA
PATRAS
LARISA
PATRAS
PATRAS
PATRAS
ATHENS
PATRAS
LARISA
LARISA
PATRAS
PATRAS
PATRAS

PFGE TYPE ST

$1 \quad 121$

12121

$1 \quad 121$

$1 \quad 121$

$1 \quad 121$

$1 \quad 121$

$1 \quad 121$

$1 \quad 121$

$1 \quad 121$

$1 \quad 121$

$1 \quad 121$

$\begin{array}{ll}1 & 121\end{array}$

$1 \quad 121$

$1 \quad 121$

$4 \quad 21$

21

Fig. 4 Dendrogram of representative mupirocin-resistant MSSA strains 
PFGE typing revealed a mostly unvarying population; the $260 \mathrm{~S}$. aureus were grouped into three PFGE types. One major pulsotype (type 1) was identified comprising of 258/260 (99.2\%) tested strains. MLST revealed that this pulsotype was classified to sequence type ST121. One isolate recovered from a patient with skin lesions was classified as PFGE type 2, ST1. Another S. aureus strain, also from skin lesions, belonged to PFGE type 4, ST21 (Fig. 4). ST1 is the primary group founder of clonal complex 1 and ST21 belongs to clonal complex 22.

The majority of the 260 tested isolates carried eta (244, 93.85\%), etb (255, $98.08 \%)$ and $231(88.85 \%)$ also carried $f n b A$. From the 258 isolates which belonged to ST121, 242/258 (93.8\%) carried both eta and etb, two $(0.78 \%)$ carried only eta, whereas, another 13/258 (5.04\%) were only $e t b$-positive; nine among them carried the PVL-encoding genes, all isolated in PAKCH during 2014-2016. One ST121 strain was negative for exfoliative and PVL toxin genes. Most ST121 S. aureus $(88.76 \%)$ carried also fnbA. The unique strain classified as ST1 was PVL-and fnbA-positive but negative for the exfoliative toxin genes, while the ST21 strain was negative for all the toxin genes tested. In Table 2 the molecularly analyzed isolates are presented according to patients' age and clinical entities.

\section{Discussion}

In this study, S. aureus recovered from patients with SSTIs among three tertiary-care hospitals in Greece were analyzed in terms of antimicrobial resistance and genetic diversity. S. aureus was the main etiologic agent of SSTIs during the five-year period, isolated from $46.4 \%$ of the cases, whereas, the majority was MSSA (78.2\%). Studies worldwide have demonstrated the virulence of $S$. aureus and its association with SSTIs $[1,2,5,8-10,14]$. Determination of clonal distribution and virulence factors of community-acquired S. aureus clinical isolates from purulent SSTIs in Beijing, China showed MSSA prevalence (79.6\%), in agreement to our results [15].

The increasing resistance of staphylococci to antimicrobials is also well described. Approximately onethird of S. aureus from SSTIs and nasal colonization from patients within 10 primary care clinics in South Texas, USA, were multi-drug resistant, especially isolates causing SSTIs as compared to nasal colonizers (37\% versus $11 \%$, respectively) [16]. In our study, the majority of $S$. aureus even though MSSA, were multiresistant, expressing resistance to penicillin, mupirocin, fusidic acid (92.7\%), tobramycin (88.9\%) and $21.1 \%$ to macrolides.

Table 2 Clonal and toxin distribution of the molecularly analyzed strains in relation to patients' age and clinical entities

\begin{tabular}{|c|c|c|c|c|c|c|}
\hline & Clinical entities & Clones & $P V L$ & eta & etb & $f n b A$ \\
\hline \multirow[t]{13}{*}{ Children (181) } & Nonbullous impetigo (55) & ST121 (55) & - & 54 & 53 & 55 \\
\hline & Bullous impetigo (35) & ST121 (35) & - & 34 & 35 & 33 \\
\hline & Nasal (28) & ST121 (28) & - & 26 & 28 & 27 \\
\hline & SSSS (13) & ST121 (13) & - & 13 & 13 & 12 \\
\hline & Paronychia (11) & ST121 (11) & - & 9 & 11 & 9 \\
\hline & Furunculosis (8) & ST121 (8) & - & 6 & 8 & 8 \\
\hline & Abscess (8) & ST121 (8) & 7 & 7 & 8 & 6 \\
\hline & Infected dermatitis (7) & ST121 (7) & - & 6 & 7 & 6 \\
\hline & Otorrhea (5) & ST121 (5) & - & 5 & 5 & 4 \\
\hline & Ophthalmia (4) & ST121 (4) & - & 4 & 4 & 3 \\
\hline & Vaginitis (3) & ST121 (3) & - & - & 2 & 2 \\
\hline & Omphalitis (3) & ST121 (3) & - & 3 & 3 & 2 \\
\hline & Cellulitis (1) & ST121 (1) & - & 1 & 1 & 1 \\
\hline \multirow[t]{8}{*}{ Adults (79) } & Infected dermatitis (27) & ST121 (26) & - & 26 & 26 & 23 \\
\hline & & ST21 (1) & - & - & - & - \\
\hline & Paronychia (27) & ST121 (27) & - & 26 & 27 & 20 \\
\hline & Furunculosis (20) & ST121 (20) & 1 & 20 & 20 & 17 \\
\hline & Abscess (2) & ST121 (1) & 1 & 1 & 1 & - \\
\hline & & ST1 (1) & 1 & - & - & 1 \\
\hline & Nasal (2) & ST121 (2) & - & 2 & 2 & 2 \\
\hline & Mastitis (1) & ST121 (1) & - & 1 & 1 & - \\
\hline Total (260) & & & 10 & 244 & 255 & 231 \\
\hline
\end{tabular}


The resistance of staphylococcal isolates to mupirocin in Greece was low in 2002 (2\%) [17] and it is increasing from 2010; a six-year (2010-2015) retrospective review of $S$. aureus skin infections in Athens' hospitals showed that $11.4 \%$ of MSSA were resistant to mupirocin [18]. In 2014, ST121 mupirocin-resistant clone has emerged among children attending $\mathrm{PAKCH}$, in Athens [14]. In the present multi-centre study, mupirocin, fusidic acid, tobramycin and macrolide resistance was associated with mupA, fusB, ant(4')-Ia and erm carriage, respectively. In our tertiary care centers, an annual increase of mupirocin-resistant MSSA recovered from patients with SSTIs was observed from 2014 to 2017 , with a slight decrease in 2018. Overall, the resistance to mupirocin was $30.88 \%$ among MSSA in SSTIs-associated isolates. Two mechanisms of high-level mupirocin resistance have been detected; the first one is mediated by acquisition of a plasmid-mediated mupA or ileS2 gene, whereas, the second is due to $\operatorname{mupB}$ gene (showing $65 \%$ similarity with $m u p A)$. Mupirocin resistance has been increasing among staphylococci in many parts of the world and it seems to be more common among MRSA due to prior mupirocin use $[14,19]$. Resistance to fusidic acid due to the presence of fusB is highly indicative of ST121 clonal spread, as it was firstly detected in PAKCH [14].

Aminoglycosides' resistance rate and AMEs gene presence in $S$. aureus may be due to the predominance of specific clones but also to aminoglycosides' usage in each area combined with a possible horizontal AMEs gene transfer, as reported in a previous Greek study, where aph(3')-IIIa predominated [20]. Therefore, the detection of tobramycin-resistant/gentamicin-susceptible isolates carrying ant (4')-Ia gene in the present study, besides clonal spread, may also reflect aminoglycosides' usage.

The prevalence of erm $C$ has been previously reported in Greece among macrolide-resistant staphylococci, whereas, the rate of erythromycin resistance was statistically significantly lower in MSSA (20.7\%) than in MRSA (58.6\%) and similar to our findings $(21.1 \%)$ [20, 21].

Fibronectin-binding protein A (FnBPA) mediates adhesion of $S$. aureus to fibrinogen, elastin and fibronectin, favoring the establishment of staphylococcal infections. In a study from Turkey, $f n b A$ was detected in $77.7 \%$ of strains from wound specimens, a lower percentage as compared to our study (88.85\%) [22].

Exfoliative toxins are implicated in impetigo, a common superficial skin infection in children and rarely in the development of the staphylococcal-scalded skin syndrome (SSSS), also known as Ritter disease, a generalized blistering skin disorder. The condition is mostly observed in neonates and children younger than 5 years with a peak between 2 and 3 years of age [23]. The majority of our 260 molecularly tested isolates carried eta
(93.85\%) and etb (98.08\%) but only 10/260 tested isolates were PVL-positive. In an epidemiological study among MRSA in Greece over a 12-year period (20012012), an increasing rate of MRSA among S. aureus infections was detected up to 2008, encoding mainly PVL (63.85\%) [2]. Similarly to our findings, in a collection of pediatric MSSA from SSTIs over a 43month period in Athens, Greece, $93 \%$ of isolates carried both eta and etb [14]. In a collection of S. aureus from SSTIs among pediatric patients referred to the Children's Medical Center Hospital in Tehran, during one-year period (2017-2018), eta was the most prevalent gene with a high occurrence $(100 \%$ in both MRSA and MSSA) and etb was found in only $23.8 \%$ of the MSSA population, whereas in our study etb was the predominant toxins' gene in MSSA [24]. It is remarkable that almost all staphylococcal strains harbor both the eta and etb genes, in accordance with the study conducted in a single center (PAKCH) [14], whereas only 0.5 to $3 \%$ of MSSA strains examined in previous studies carried those genes [25].

Regarding clonal dissemination in the present study, the vast majority of the molecularly analysed strains belonged to a single clone, ST121, which has been identified in several studies worldwide [14, 26-31]. The emergence of this predominant MSSA clone, resistant to mupirocin and highly resistant to tobramycin and fusidic acid is associated with a sharp increase in SSSS cases in Greece since 2015, with 31 cases firstly documented in a children's hospital in Athens during 2014-2017 [14, 26]. Presumably, the indiscriminate and repetitive use of mupirocin as an over-the-counter agent, drives the selection of this clone, as shown in a previous work from pediatric mupirocin-resistant isolates where $32.3 \%$ of patients reported past use of topical antimicrobials, with $20.6 \%$ of lesions being recurrent [14].

ST121 was identified in several countries among $S$. aureus causing SSTIs, such as, Paraguay, New Caledonia, Togo, France, Czech Republic, Germany, Turkey, US, Fr. West Indies, UK, Polynesia, Switzerland, Spain, Algeria, The Netherlands and China showing an overall prevalence rate approximately $10 \%$ in MSSA, and less than $5 \%$ in MRSA [27, 28]. In Portugal, in a study among children with SSTIs, $63 \%$ of S. aureus belonged to ST121 [29]. In Yangon, Myanmar, ST121 was found among pvl-positive MSSA, in contrast to our MSSA collection [30]. In agreement to our results, in Houston, Texas, $S$. aureus recovered from children with SSSS revealed that 40/58 isolates belonged to ST121, while 11 more strains belonged to STs from the same Clonal Complex (CC121), the majority being MSSA carrying eta and $e t b$ [31].

In the present collection of strains, only 10/260 S. aureus were PVL-positive. This is associated with the vast 
predominance of ST121 carrying mainly eta/etb genes, over other MSSA clones (ST1 and ST21). ST1 has been reported as one of the main clones among MSSA in a study from Guangzhou, China [32]. An investigation on the global population structure of PVL-positive MSSA from five continents up to 2007, revealed that the most frequent STs were ST30 (19.9\%) and ST121 (19.9\%) [33]. In our study only $2 / 260$ of strains were classified in other sequence types; one strain carrying only PVL was identified as ST1 and another strain, negative for all the toxins' genes tested, as ST21. In a previous Greek study, we found that $45 \%$ of MRSA and only $12 \%$ of MSSA carried the PVL genes [34]. Still, MSSA populations can be PVL-positive in majority, as reported by Darboe et al., among Gambian patients with invasive and noninvasive infections, where PVL-positive strains accounted for $61.4 \%(180 / 293)$ of S. aureus isolates [35].

A limitation of the present study is that the great majority of patients were admitted at the Departments for outpatients and therefore no data were available about any therapeutic failures or alternative treatments.

\section{Conclusions}

The dominant ST121 clone in our study, associated with high-level resistance to mupirocin, prevailed among mupirocin-resistant staphylococcal isolates from SSTIs. This successful clone, comprised of strains carrying resistance determinants for topical antimicrobials, exfoliative toxins and adhesin genes, affects adult and pediatric patients in three different areas of Greece during the five-year period. Clinicians should be aware of this clone whereas, there is need for de-escalation of mupirocin use in the community.

\section{Methods}

\section{Patients and hospitals}

A total of $6145 \mathrm{~S}$. aureus were recovered during a fiveyear period (2014-2018) from 13,244 patients with SSTIs admitted in three tertiary-care hospitals in different areas of Greece, serving about one third of the population; the University General Hospital of Patras (UGHP: 2579 isolates, 770 beds, with approximately 37,000 admissions per year in Southwestern Greece), P. \& A. Kyriakou Children's Hospital in Athens that is a tertiary care referral center for children (PAKCH: 1964 isolates, 400 beds, 16,000 admissions) and the University General Hospital of Larissa (UGHL: 1602 isolates, 650 beds, 35, 000 admissions, in Central Greece). Children's hospitals admit patients up to the age of 16 years.

Skin infections were classified as secondarily infected dermatitis, impetigo (bullous and nonbullous), furunculosis or scalded skin syndrome. Soft tissue infections such as cellulitis and abscess, otorrhea, ophthalmia, vaginitis, omphalitis, mastitis, and paronychia were also included in the study. Staphylococcal strains isolated from nasal cultures were included in the analysis only if skin lesions were clinically relevant (e.g., multiple skin lesions). The Ethics Committees of the UGHP, PAKCH and UGHL approved this study and waived the need for informed consent (Approval Numbers: 786, 9956 and 1355 , respectively).

\section{Phenotypic identification and antibiotic susceptibility testing}

Staphylococci were identified to species level based on colony morphology, Gram staining, catalase production, coagulase testing (Slidex Staph Plus; bioMérieux, Marcy l' Etoile, France) and by the Vitek2 System (GP card, bioMerieux). Susceptibility to cefoxitin (FOX), penicillin (PEN), erythromycin (ER), clindamycin (CLI), tobramycin (TOB), gentamicin (GM), ciprofloxacin (CIP), fusidic acid (FA), rifampicin (RIF), tetracycline (TET) and sulfamethoxazole/ trimethoprim (SXT), was tested by the disk diffusion method according to EUCAST guidelines [36]. Testing for inducible and constitutive lincosamide resistance was performed with the D-test. MICs of mupirocin (MUP) and oxacillin (OX) were determined by $\mathrm{E}^{- \text {test }^{\circ}}$ (bioMerieux). Isolates resistant to at least three classes of antimicrobials were considered multidrug resistant. Phenotypic determination of MSSA was based on cefoxitin disk susceptibility [36]. Isolates with mupirocin MIC $>1 \mathrm{mg} / \mathrm{L}$ were further analysed.

\section{Molecular analysis}

Two hundred and sixty representative mupirocinresistant isolates were sent to the National Reference Laboratory for Staphylococci for molecular analysis; 110 isolates from the UGHP, 47 isolates from the UGHL and 103 isolates from the PAKCH. Strains were selected according to the sample size of participating hospitals, age and gender of patients, clinical specimen and date of sampling, infection site, clinical entities, hospital ward, and whether the isolate was epidemiologically representative of a cluster, including colonizing ones.

Amplification of genes encoding Panton-Valentine Leukocidin (PVL, lukS/lukF-PV), exfoliative toxins (eta, $e t b)$, adhesin FnbA (fnbA) and the resistance genes mupA (encoding high-level resistance to mupirocin), fusB (fusidic acid), ermA, ermC and msrA (macrolides/

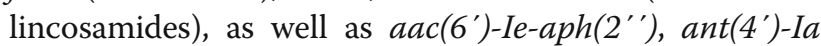
and aph (3')-IIIa encoding aminoglycosides' modifying enzymes (AMEs), was performed by PCRs with specific primers, as described. PCR products were analyzed by electrophoresis on $1 \%$ agarose gels. S. aureus strains ATCC 49,775 (PVL-positive), Fri913 (fnbA-positive), A920211 (eta/etb-positive, mupA-positive), were used as controls for the PCRs [20, 21, 37-40]. 


\section{Clonal identification}

From the 260 representative strains, DNA extraction was performed into agarose disks and strains were classified into pulsotypes by pulsed-field gel electrophoresis (PFGE) of chromosomal DNA SmaI digests performed in a CHEF DR III apparatus (Bio-Rad, Richmond, CA), as previously described [41]. A dendrogram comparing molecular weights of strains' DNA fragments was performed by FPQuest software version 4.5 (Bio-Rad Laboratories Inc). According to criteria established by Miragaia et al. [42] patterns differing by less than $79 \%$ (corresponding to a difference of less than seven bands) were considered to belong to the same PFGE type.

The selected $S$. aureus strains were also characterized by Multilocus Sequence Typing (MLST) (http://mlst.net) [43]. The results were analyzed by the application of eBURST algorithm. Clonal complexes were defined by using the default setting, in which all STs within a clonal complex differ by no more than one allele from at least one other ST in the clonal complex.

\section{Statistical analysis}

Counts and percentages are reported for categorical variables. Statistical analysis was performed using SPSS for Windows (version 18; SPSS, Chicago, IL).

\section{Abbreviations}

AMEs: Aminoglycosides' modifying enzymes; CIP: Ciprofloxacin;

CLI: Clindamycin; ER: Erythromycin; EUCAST: European Committee on Antimicrobial Susceptibility Testing; FA: Fusidic acid; FnBPA: Fibronectinbinding protein A; FOX: Cefoxitin; GM: Gentamicin; MIC: Minimum inhibitory concentration; MLST: Multilocus Sequence Typing; MSCRAMMs: Microbial Surface Components Recognizing Adhesive Matrix Molecules; MRSA: Methicillin-resistant Staphylococcus aureus; MSSA: Methicillinsusceptible Staphylococcus aureus; MUP: Mupirocin; OX: Oxacillin; PAKCH: P. \& A. Kyriakou Children's Hospital; PCR: Polymerase chain reaction; PEN: Penicillin; PFGE: Pulsed-field gel electrophoresis; PVL: Panton-Valentine leukocidin; RIF: Rifampicin; SPSS: Superior Performance Software System; SSSS: Staphylococcal scalded skin syndrome; SSTIs: Skin and soft tissue infections; ST: Sequence type; SXT: Sulfamethoxazole/ trimethoprim; TET: Tetracycline; TOB: Tobramycin; UGHL: University General Hospital of Larissa; UGHP: University General Hospital of Patras

\section{Supplementary Information}

The online version contains supplementary material available at https://doi. org/10.1186/s12866-021-02272-5.

Additional file 1: Supplementary Figure 1: PFGE of S. aureus after DNA digestion with Smal.

\section{Acknowledgements}

Not applicable.

\section{Authors' contributions}

All authors contributed to the study conception and design. Material preparation, data collection and analysis were performed by NG, $A D, K T, M M$, GK, VS and FK. Molecular analysis was performed by MM and NG. The first draft of the manuscript was written by NG and all authors commented on previous versions of the manuscript. Supervision of the study, as well as review and editing of the manuscript were performed by EP, EL and IS. All authors read and approved the final manuscript.

\section{Funding}

This study was supported by funds of the participating laboratories; funding of the Research Committee of University of Thessaly, Greece, Grant number 3811.1 under the scientific responsibility of E.P and funding of the Research Committee of the University of Patras, Greece, Grant number 39,540,000 under the scientific responsibility of IS.

\section{Availability of data and materials}

The datasets used and/or analyzed during the current study are available from the corresponding author on reasonable request.

\section{Declarations}

Ethics approval and consent to participate

The Ethics Committees of the UGHP, PAKCH and UGHL approved this study and waived the need for informed consent (Approval Numbers: 786, 9956 and 1355 , respectively).

\section{Consent for publication}

Not applicable.

Competing interests

The authors declare that they have no competing interests.

\section{Author details}

${ }^{1}$ Department of Microbiology, School of Medicine, University of Patras, 26504 Patras, Greece. ${ }^{2}$ National Reference Laboratory for Staphylococci, University of Patras, Patras, Greece. ${ }^{3}$ Department of Microbiology P. \& A, Kyriakou Children's Hospital, Athens, Greece. ${ }^{4}$ Department of Microbiology, School of Medicine, University of Thessaly, Larissa, Greece. ${ }^{5}$ Department of Microbiology, University General Hospital of Patras, Patras, Greece.

Received: 2 April 2021 Accepted: 13 June 2021

Published online: 03 July 2021

\section{References}

1. Sakr A, Brégeon F, MègeJL, Rolain JM, Blin O. Staphylococcus aureus Nasal Colonization: An Update on Mechanisms, Epidemiology, Risk Factors, and Subsequent Infections. Front Microbiol. 2018;9:2419. https://doi.org/10.3389/ fmicb.2018.02419.

2. Drougka E, Foka A, Liakopoulos A et al. A 12-year survey of methicillinresistant Staphylococcus aureus infections in Greece: ST80-IV epidemic? Clin Microbiol Infect 2014:20: 0796-803.

3. Foster TJ. The MSCRAMM Family of Cell-Wall-Anchored Surface Proteins of Gram-Positive Cocci. Trends Microbiol. 2019;27(11):927-41. https://doi.org/1 0.1016/j.tim.2019.06.007 Epub 2019 Jul 30.

4. Haveri M, Hovinen M, Roslof A, Pyorala S. Molecular types and genetic profiles of Staphylococcus aureus strains isolated from bovine intramammary infections and extramammary sites. J Clin Microbiol 2008;46: 3728-3735.

5. Edwards AM, Potts JR, Josefsson E, et al. Staphylococcus aureus host cell invasion and virulence in sepsis is facilitated by the multiple repeats within FnBPA. PLoS Pathog. 2010;6:e1000964.

6. Ghasemian A, Najar Peerayeh S, Bakhshi B, Mirzaee M. The Microbial Surface Components Recognizing Adhesive Matrix Molecules (MSCRAMMs) Genes among Clinical Isolates of Staphylococcus aureus from Hospitalized Children. Iran J Pathol. 2015;10:258-64.

7. Vazquez-Rosas GJ, Merida-Vieyra J, Aparicio-Ozores G, Lara-Hernandez A, De Colsa A, Aquino-Andrade A. Molecular Characterization of Staphylococcus aureus Obtained from Blood Cultures of Paediatric Patients Treated in a Tertiary Care Hospital in Mexico. Infect Drug Resist. 2021;14:1545-56.

8. Tromp AT, van Strijp JAG. Studying Staphylococcal Leukocidins: a Challenging Endeavor. Front Microbiol. 2020;11:611. https://doi.org/10.3389/ fmicb.2020.00611 eCollection 2020.

9. Oliveira D, Borges A, Simões M. Staphylococcus aureus Toxins and Their Molecular Activity in Infectious Diseases. Toxins (Basel). 2018;10(6):E252. https://doi.org/10.3390/toxins10060252.

10. Tong SY, Davis JS, Eichenberger E, Holland TL, Fowler VG Jr. Staphylococcus aureus infections: epidemiology, pathophysiology, clinical manifestations, and management. Clin Microbiol Rev. 2015;28(3):603-61. https://doi.org/1 0.1128/CMR.00134-14 
11. Vestergaard M, Frees $D$, Ingmer $H$. Antibiotic Resistance and the MRSA Problem. Microbiol Spectr 2019;7(2). https://doi.org/10.1128/microbiolspec. GPP3-0057-2018.

12. Mantle P. Nasal Decolonisation of MRSA. Antibiotics (Basel). 2019;8(1):14. https://doi.org/10.3390/antibiotics8010014 Published 2019 Feb 4.

13. Tucaliuc A, Blaga AC, Galaction Al, Cascaval D. Mupirocin: applications and production. Biotechnol Lett 2019;41:495-502. https://doi.org/10.1007/s1052 9-019-02670-w

14. Doudoulakakis A, Spiliopoulou I, Spyridis N, et al. Emergence of a Staphylococcus aureus Clone Resistant to Mupirocin and Fusidic Acid Carrying Exotoxin Genes and Causing Mainly Skin Infections. J Clin Microbiol. 2017;55(8):2529-37. https://doi.org/10.1128/JCM.00406-17.

15. Xiao N, Yang J, Duan N, Lu B, Wang L. Community-associated Staphylococcus aureus $\mathrm{PVL}^{+}$ST22 predominates in skin and soft tissue infections in Beijing, China. Infect Drug Resist. 2019;12:2495-503. https://doi. org/10.2147/IDR.S212358.

16. Lee GC, Dallas SD, Wang Y, et al. Emerging multidrug resistance in community-associated Staphylococcus aureus involved in skin and soft tissue infections and nasal colonization. J Antimicrob Chemother. 2017;72(9):24618. https://doi.org/10.1093/jac/dkx200.

17. Petinaki E, Spiliopoulou I, Kontos F et al. Clonal dissemination of mupirocinresistant staphylococci in Greek hospitals. J Antimicrob Chemother 2004;53: 105-108. https://doi.org/10.1093/jac/dkh028.

18. Stefanaki C, leronymaki A, Matoula T, et al. Six-Year Retrospective Review of Hospital Data on Antimicrobial Resistance Profile of Staphylococcus aureus Isolated from Skin Infections from a Single Institution in Greece. Antibiotics (Basel). 2017:6(4):39. https://doi.org/10.3390/antibiotics6040039.

19. Khoshnood S, Heidary M, Asadi $A$, et al. A review on mechanism of action, resistance, synergism, and clinical implications of mupirocin against Staphylococcus aureus. Biomed Pharmacother. 2019;109:1809-18. https://doi. org/10.1016/j.biopha.2018.10.131.

20. Sarrou S, Malli E, Tsilipounidaki K, et al. MLSB-Resistant Staphylococcus aureus in Central Greece: Rate of Resistance and Molecular Characterization. Microb Drug Resist. 2019;25(4):543-50. https://doi.org/10.1089/mdr.2018.0259 Epub 2018 Nov 7.

21. Spiliopoulou I, Petinaki E, Papandreou P, Dimitracopoulos G. erm (C) is the predominant genetic determinant for the expression of resistance to macrolides among methicillin-resistant Staphylococcus aureus clinical isolates in Greece. J Antimicrob Chemother 2004;53(5):814-817.https://doi. org/10.1093/jac/dkh197.

22. Demir C, Demirci M, Yigin A, Tokman HB, CetikYildiz S. Presence of biofilm and adhesin genes in Staphylococcus aureus strains taken from chronic wound infections and their genotypic and phenotypic antimicrobial sensitivity patterns. Photodiagnosis Photodyn Ther. 2020;29:101584. https:// doi.org/10.1016/j.pdpdt.2019.101584.

23. Leung AKC, Barankin B, Leong KF. Staphylococcal-scalded skin syndrome: evaluation, diagnosis, and management. World J Pediatr. 2018;14(2):116-20. https://doi.org/10.1007/s12519-018-0150-x.

24. Maleki DT, Ghalavand Z, Laabei M, et al. Molecular analysis of accessory gene regulator functionality and virulence genes in Staphylococcus aureus derived from pediatric wound infections. Infect Genet Evol. 2019;73:255-60. https://doi.org/10.1016/j.meegid.2019.05.013.

25. Grumann D, Nubel U, Broker BM. Staphylococcus aureus toxins: their functions and genetics. Infect Genet Evol 2014;21:583-592. https://doi.org/1 0.1016/.j.meegid.2013.03.013.

26. Doudoulakakis A, Spiliopoulou I, Syridou G, et al. Emergence of staphylococcal scalded skin syndrome associated with a new toxinogenic, methicillin-susceptible Staphylococcus aureus clone. J Med Microbiol. 2019; 68(1):48-51. https://doi.org/10.1099/jmm.0.000871.

27. Rao Q, Shang W, Hu X, Rao X. Staphylococcus aureus ST121: a globally disseminated hypervirulent clone. J Med Microbiol. 2015;64(12):1462-73. https://doi.org/10.1099/jmm.0.000185.

28. Jiang W, Zhou Z, Zhang K, Yu Y. Epidemiological investigation of community-acquired Staphylococcus aureus infection. Genet Mol Res. 2013; 12(4):6923-30. https://doi.org/10.4238/2013 December.19.11.

29. Conceição T, Aires-de-Sousa M, Pona N, et al. High prevalence of ST121 in community-associated methicillin-susceptible Staphylococcus aureus lineages responsible for skin and soft tissue infections in Portuguese children. Eur J Clin Microbiol Infect Dis. 2011;30(2):293-7. https://doi.org/10.1 007/s10096-010-1087-8.
30. Aung MS, Urushibara N, Kawaguchiya M, et al. Virulence factors and genetic characteristics of methicillin-resistant and -susceptible Staphylococcus aureus isolates in Myanmar. Microb Drug Resist. 2011;17(4):525-35. https://doi.org/1 0.1089/mdr.2011.0061.

31. Hultén KG, Kok M, King KE, LamberthLB, Kaplan SL. Increasing Numbers of Staphylococcal Scalded Skin Syndrome Cases Caused by ST121 in Houston, Texas. Pediatr Infect Dis J. 2020;39(1):30-4. https://doi.org/10.1097/INF. 0000000000002499

32. Liang B, Mai J, Liu Y, et al. Prevalence and Characterization of Staphylococcus aureus Isolated From Women and Children in Guangzhou, China. Front Microbiol. 2018;9:2790. https://doi.org/10.3389/fmicb.2018.02 790.

33. Rasigade JP, Laurent F, Lina G et al. Global distribution and evolution of Panton-Valentine leukocidin-positive methicillin-susceptible Staphylococcus aureus, 1981-2007. J Infect Dis 2010:201:1589-1597

34. Chini V, Petinaki E, Foka A, Paratiras S, Dimitracopoulos G. Spiliopoulou. Spread of Staphylococcus aureus clinical isolates carrying Panton-Valentine leukocidin genes during a 3-year period in Greece. Clin Microbiol Infect. 2006;12(1):29-34. https://doi.org/10.1111/j.1469-0691.2005.01295.x.

35. Darboe S, Dobreniecki S, Jarju S, et al. Prevalence of Panton-Valentine Leukocidin (PVL) and Antimicrobial Resistance in Community-Acquired Clinical Staphylococcus aureus in an Urban Gambian Hospital: A 11-Year Period Retrospective Pilot Study. Front Cell Infect Microbiol. 2019;9:170. https://doi.org/10.3389/fcimb.2019.00170.

36. The European Committee on Antimicrobial Susceptibility Testing Breakpoint tables for interpretation of MICs and zone diameters. 2018, v_8.1 http:// www.eucast.org

37. Jarraud S, Mougel C, Thioulouse J et al. Relationships between Staphylococcus aureus genetic background, virulence factors, agr groups (alleles), and human disease. Infect Immun 2002;70 (2), 631-641.

38. Gomes AR, Vinga S, Zavolan M, de Lencastre $H$. Analysis of the genetic variability of virulence-related loci in epidemic clones of methicillin-resistant Staphylococcus aureus. Antimicrob Agents Chemother 2005;49: 366-379.

39. Hung WC, Chen HJ, Lin YT et al. Skin commensal staphylococci may act as reservoir for fusidic acid resistance genes. PLoS One 2015;10:e0143106. https://doi.org/10.1371/journal.pone.0143106.

40. Liakopoulos A, Foka A, Vourli S, et al. Aminoglycoside-resistant staphylococc in Greece: prevalence and resistance mechanisms. Eur J Clin Microbiol Infect Dis. 2011;30:701-5. https://doi.org/10.1007/s10096-010-1132-7.

41. Tenover FC,Arbeit RD, Goering RV et al. Interpreting chromosomal DNA restriction patterns produced by pulsed-field gel electrophoresis: Criteria for bacterial strain typing. J Clin Microbiol 1995;33: 2233-2239.

42. Miragaia M, Carrico JA, Thomas JC, Couto I, Enright MC, de Lencastre H. Comparison of molecular typing methods for characterization of Staphylococcus epidermidis: Proposal for clone definition. J Clin Microbiol 2008:46: 118-129.

43. Thomas JC, Vargas MR, Miragaia M, Peacock SJ, Archer GL, Enright MC. Improved multilocus sequence typing scheme for Staphylococcus epidermidis. J Clin Microbiol 2007;45: 616-619.

\section{Publisher's Note}

Springer Nature remains neutral with regard to jurisdictional claims in published maps and institutional affiliations.

Ready to submit your research? Choose BMC and benefit from:

- fast, convenient online submission

- thorough peer review by experienced researchers in your field

- rapid publication on acceptance

- support for research data, including large and complex data types

- gold Open Access which fosters wider collaboration and increased citations

- maximum visibility for your research: over $100 \mathrm{M}$ website views per year

At $\mathrm{BMC}$, research is always in progress.

Learn more biomedcentral.com/submissions 